\title{
Cell density-dependent regulation of $p 73$ in breast cancer cells
}

\author{
CHAITALI TOPHKHANE, SHIHE YANG, ZHIZHUANG JOE ZHAO and XIAOHE YANG
}

Department of Pathology, University of Oklahoma Health Sciences Center; Oklahoma City, OK 73104, USA

Received July 30, 2009; Accepted August 27, 2009

DOI: 10.3892/ijo_00000461

\begin{abstract}
Molecular regulation of p73, a p53 family member, remains unclear. Here we report that p73 expression is significantly regulated by cell densities. In particular, we found that $\mathrm{p} 73 \alpha$ and $\mathrm{p} 73 \beta$ are differentially regulated. While $\mathrm{p} 73 \beta$ protein levels were inversely correlated with cell densities, p73 $\alpha$ protein levels behaved oppositely. We further showed that density-dependent changes of p $73 \alpha$ follow the same patterns as E2F-1 and TAp73 mRNA levels, suggesting transcriptional regulation. Our data also suggest that high levels of p73ß at lower densities may be due to increased protein stability. However, AIP-4/Itch appeared not to be involved in downregulation of p73ß at high densities. Moreover, we also found that subcellular location of p73 isoforms changes with the culture density increases. While high level of p $73 \beta$ at low density was mainly presented in the nucleus, low levels of this protein at high densities were mainly in the cytosol. Taken together, these findings reveal a novel mechanism that differentially regulates p73 isoforms and underscores the role of cell-cell interaction in p73 regulation, which may advance our understanding of p73 expression and function in human cancers.
\end{abstract}

\section{Introduction}

p73 is a member of the p53 family of transcription factors, which is implicated in cell differentiation, development, apoptosis and tumor suppression (1). The p73 gene encodes two groups of isoforms: TAp 73 s and $\Delta N p 73$ s. TAp 73 s contain a transactivation (TA) domain in their N-termini, while $\Delta \mathrm{Np} 73 \mathrm{~s}$ have the TA domain missing. Alternative splicing at the carboxyl terminus of both TAp73 and $\Delta \mathrm{Np} 73$ yields further p73 isoforms. For example, TAp73s include $\mathrm{p} 73 \alpha, \beta, \chi, \delta, \varepsilon, \varphi, \gamma, \eta(2)$. The major isoforms of p73 are TAp73 $\alpha$ and TAp73ß (3). In general, TAp73 and $\Delta \mathrm{Np} 73$ are functional antagonists to each other. TAp73s are able to activate the p53 pathway; $\Delta \mathrm{Np} 73 \mathrm{~s}$ function as dominant negative mutants that inactivate p53 and TAp73 (2).

Correspondence to: Dr Xiaohe Yang, Department of Pathology, University of Oklahoma Health Sciences Center, Oklahoma City, OK 73104, USA

E-mail: xiaohe-yang@ouhsc.edu

Key words: p73, cell density, p53, E2F-1, ITCH/AIP4, breast cancer
Expression and regulation of p73 in human cancer remain unclear. In contrast to frequent p53 mutation, p73 mutation in human cancer is rare (2). However, overexpression of wildtype p73 is frequently detected in different cancers, such as breast, prostate, bladder and esophageal cancers (4). Previous studies demonstrate that $\mathrm{p} 73$ can be regulated at both mRNA and protein levels by a number of factors, including DNA damage, interactions with oncoproteins and viral proteins $(5,6)$. The TAp73 promoter is known to contain binding sites for the transcription factors E2F-1, c-Myc, and c-Myb $(7,8)$. The $\Delta \mathrm{Np} 73$ promoter contains binding sites for $\mathrm{p} 53$ and TAp73 (9,10). Activation of TAp73 transcription contributes to E2F-1-induced apoptosis (11). p73 activity is also regulated through its physical interaction with a number of binding partners, including c-Abl, p300, WT1, c-Myc, MM1, MDM-2, E1A and others (12-14). In particular, when c-Abl is activated in response to DNA damage, it phosphorylates TAp73 and increases its protein stability $(5,6,15)$. Recently, it has been shown that p73 degradation is regulated by AIP4, a ubiquitin ligase (16).

Despite significant advances, more factors that regulate p73 remain to be identified. Here we show that the expression of p73 is closely regulated by cell density. Interestingly, p $73 \alpha$ and $\mathrm{p} 73 \beta$ are diffrentially regulated at different cell densities. This novel finding suggests that cell-cell interaction and signaling from the microenvironment might contribute to the regulation of p73 expression and functional modulation.

\section{Materials and methods}

Cell culture and transfection. MCF-7, MDA-MB-231 and HS578T breast cancer cell lines were purchased from the American Type Culture Collection (ATCC). The cells were cultured in DMEM/F12 medium (Sigma Co., St. Louis, MO) containing $10 \%$ fetal bovine serum (FBS). MCF-7 cell line expressing p53 siRNA was established by transfecting MCF-7 cells with plasmid encoding p53-specific siRNA (Imgenex), followed by G418 selection. G418 resistant clones were pooled for further characterization. For determining p73 levels at different culture densities, cells were inoculated into $60-\mathrm{mm}$ dishes with cell number as low as $1 \times 10^{4}$ cells/dish to as high as $3 \times 10^{6}$ cells/dish and incubated for $48 \mathrm{~h}$. The cells were then collected to examine p73 RNA and protein levels. For AIP-4 siRNA (Dharmacon RNA Technologies, Lafayette, CO) transfection, $1 \times 10^{6}$ cells were inoculated into a $60-\mathrm{mm}$ dish $24 \mathrm{~h}$ before transfection. The transfections were performed using Lipofectamine 2000 (Invitrogen) according to manufacturer's protocol. 
Sulforhodamine B (SRB) assay. Cells were inoculated in a 24 -well plate at low $(500 /$ well) or higher $(25,000 /$ well $)$ densities. Cells from each group were then fixed with $10 \%$ trichloroacetic acid (TCA) at 24 and $48 \mathrm{~h}$ after inoculation. TCA fixed cells were then stained with $0.4 \%$ SRB for $30 \mathrm{~min}$ followed by four washes. Protein-bound dye was dissolved in $10 \mathrm{mM}$ Tris base and plates were read at $565 \mathrm{nM}$ using an ELISA reader. Cell proliferation rate was calculated based on the increase in SRB absorbance between 24 and $48 \mathrm{~h}$ of the same density group ( 3 parallel samples in each group). The data were analyzed with Student's t test.

Western blotting. The cells lysate was collected at indicated time after inoculation. Protein concentration was determined using a BCA kit. Protein lysate $(50 \mu \mathrm{g})$ was separated with 12 or $15 \%$ SDS-PAGE gel and transferred to nitrocellulose membrane. The membrane was probed with a specific primary antibody at a dilution of 1:300 to 2000, followed by washing, probing with a corresponding secondary antibody and autoradiography using an ECL kit (Amersham/GE Health Care). Antibodies against p53, p73 $\alpha$ (H79), E2F-1 AIP-4/Itch and actin were purchased from Santa Cruz Biotechnology (Santa Cruz, CA). Antibody against p73ß (AB3) was from Calbiochem. Anti-p73a (C24) antibody for immunofluorescence was purchased from Vector laboratories (Burlingame, CA).

RNA extraction and RT-PCR. Total RNA was isolated from control and treated cells using RNeasy Mini Kit (Qiagen, CA). First strand cDNA synthesis was performed using $3 \mu \mathrm{g}$ total RNA and SuperscriptIII ${ }^{\mathrm{TM}}$ First Strand synthesis system (Invitrogen). mRNA levels of p73 and actin were detected with PCR using the following primers: TAp73 forward: 5'CGG GAC GGA CGC CGA TG-3'; $\triangle$ Np73 forward: 5'-CGC CTA CCA TGC TGT ACG TCG GTG-3; p73 reverse: 5'CTT GGC GAT CTG GCA GTA G-3'. Actin forward: 5'GCA CCA CAC CTT CTA CAA TGA GC-3'; actin reverse: 5'-GAC GTA GCA CAG CTT CTC CTT AAT G-3'.

\section{Results}

Cell density-dependent regulation of $p 73 \beta$ and its correlation with proliferation rate. During our study of $\mathrm{p} 73$ regulation by other factors, we observed that $\mathrm{p} 73 \beta$ protein levels varied with culture conditions. This was based on the results detected with a well established antibody (AB3) that specifically recognizes $\mathrm{p} 73 ß$ (17). To test the correlation between cell density and $\mathrm{p} 73 \beta$ expression, we examined $\mathrm{p} 73 ß$ protein levels in MCF-7 cells grown at different densities, which varied from $1 \times 10^{4}$ to $3 \times 10^{6} / 60 \mathrm{~mm}$ dish. We found that $\mathrm{p} 73 \beta$ levels were inversely correlated with cell density. MCF-7 cells cultured at very low density expressed very high protein levels of $\mathrm{p} 73 \beta$, which decreased with increasing cell density (Fig. 1A). To exclude the possibility that density regulated p73ß in MCF-7 cells is a cell line-specific phenomenon, we examined the effect of cell density on $\mathrm{p} 73 \mathrm{~B}$ expression in MDA-MB-231 and HS578T breast cancer cell lines. As shown in Fig. 1B and C, each of the cell lines exhibited similar density-dependent regulation of $\mathrm{p} 733$. These results indicate that cell density-dependent regulation of $\mathrm{p} 73 ß$ is
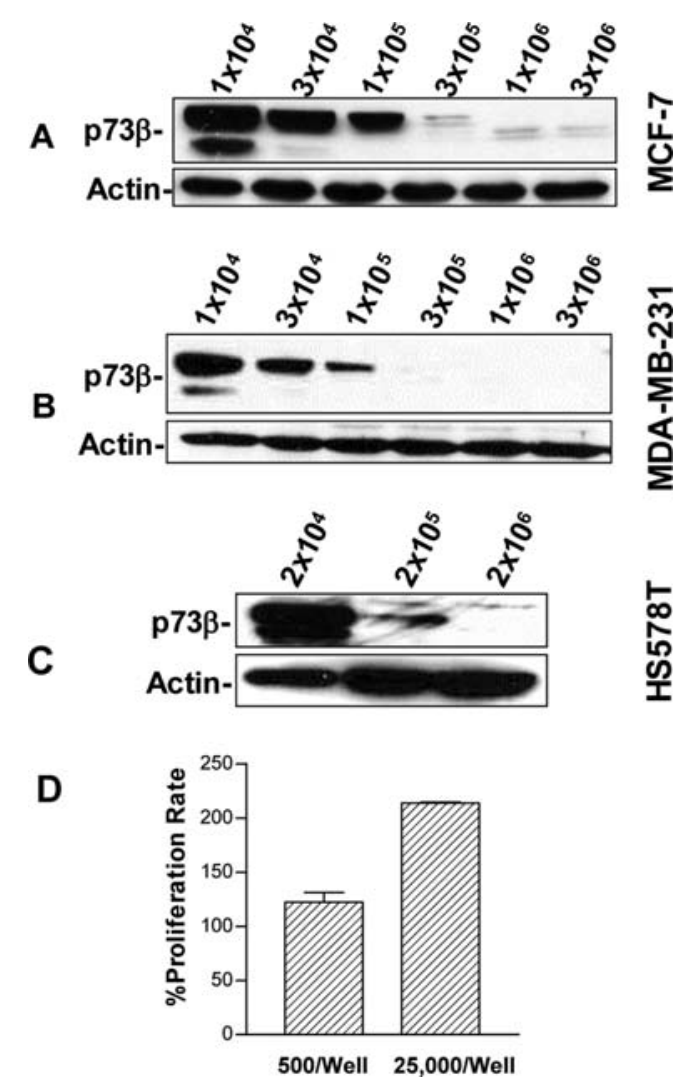

Figure 1. Cell density regulates p73 expression in breast cancer cells (A-C). MCF-7 (A), MDA-MB-231 (B) and HS578T (C) cells were seeded at different densities and cultured for $48 \mathrm{~h}$. Protein levels of p73ß were analyzed with Western blot (the p73 antibody was AB3). (D) Proliferation rate of MCF-7 cells at different densities. The cells were inoculated in a 24-well plate at 500/well or 25,000/well. Cell proliferation rate was calculated based on SRB absorbance of $48 \mathrm{~h}$ over $24 \mathrm{~h}$. *p $<0.01$.

universal. To correlate $\mathrm{p} 73 ß$ expression with cell proliferation, the proliferation rate of MCF-7 cells at low or higher densities was measured using SRB assays. As shown in Fig. 1D, cell proliferation rate at lower density (500/well of 24-well plate) was significantly lower than the higher density group $(25,000 /$ well $)$, suggesting a correlation between high p $73 ß$ levels and slower cell growth at low density.

Cell density-dependent regulation of p73a, p53 and E2F-1. Based on cell density-dependent changes in p $73 \beta$ levels, it is reasonable to question whether p73/p53 family members and their regulatory factors are modulated concomitantly. We therefore examined the protein levels of p $73 \alpha$, p53 and E2F-1 in MCF-7 cells at different densities. Of note, $\mathrm{p} 73 \alpha$ protein was identified based on a $73 \mathrm{Kd}$ band recognized by a wellestablished p73 antibody, H79 (18). As shown in Fig. 2A, we found that the expression of all these factors was regulated by cell density, although to a lesser extent. In contrast to p73ß, protein levels of p73 $\alpha$, p53 and E2F-1 were lower at low density but higher in high density. These results indicate that the pattern of cell density-dependent regulation of $\mathrm{p} 73 \alpha$, p53 and E2F-1 was opposite to that of p73ß.

Previous studies suggest that expression of both TAp73 and $\Delta \mathrm{Np} 73$ expression can be regulated by p53 $(19,20)$. Our results show that, in cell density-related regulation, $\mathrm{p} 53$ levels 

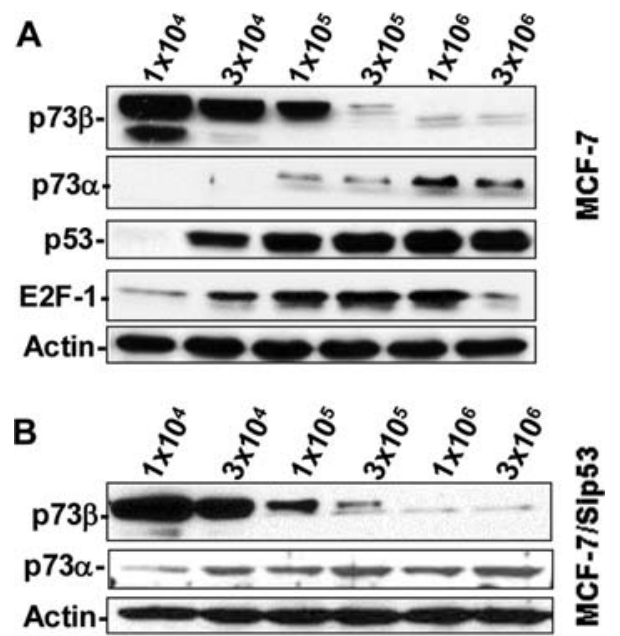

Figure 2. (A) Regulation of p53, p73 family of proteins by cell density. MCF-7 cells were inoculated at increasing cell densities and cultured for $48 \mathrm{~h}$ Protein levels of individual markers were detected suing Western blot with anti-p73 $\alpha$ (H79), anti-p53 (DO-1), anti-E2F-1 (KH-95) and AIP4/Itch (G-11) antibodies. (B) Density-dependent regulation of p73 is independent of cellular p53 status. Control and MCF-7/p53siRNA cells were inoculated at indicated densities and 73 protein levels were determined by Western blotting with anti-p73ß antibody (AB3).

A

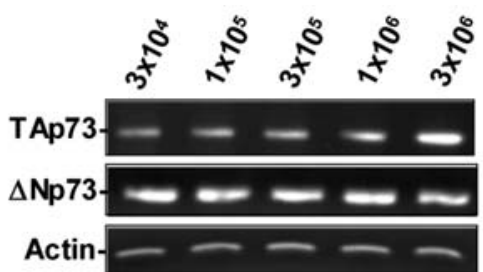

B

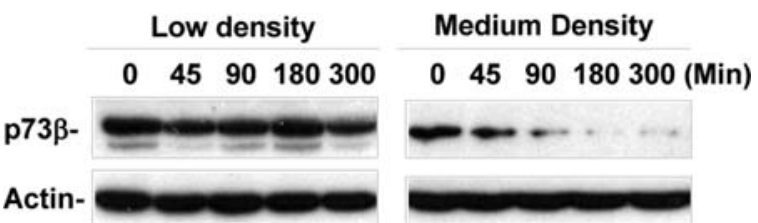

Figure 3. (A) Cell density-dependent regulation of p73 transcription. MCF-7 cells were cultured at indicated densities for $48 \mathrm{~h}$, followed by RNA extraction. TAp73 mRNA levels were detected by RT-PCR using genespecific primers. (B) p 733 protein stability is increased in low density cultures. MCF-7 cells were cultured at low $\left(2 \times 10^{4}\right)$ and medium $\left(2 \times 10^{5}\right)$ densities overnight and treated with $50 \mu \mathrm{M}$ CHX for indicated time points. Whole cell lysates were collected and subjected to Western blot analysis using anti-p73ß antibody (AB3).

were inversely correlated with p73ß but parallel to the changes in $\mathrm{p} 73 \alpha$. To test whether cell density-dependent regulation of p73 is p53-dependent, we examined the p73ß levels in MCF-7/p53siRNA cell line, which is a stable MCF-7 subline transfected with p53-specific siRNA. As shown in Fig. 2B, p73ß protein levels in MCF-7/p53siRNA cells at different densities show a similar pattern to MCF-7 cells. The results suggest that cell density-dependent regulation of $\mathrm{p} 733$ is $\mathrm{p} 53$ independent. This is supported by similar density-dependent expression of p73ß in MDA-MB-231 cells (Fig. 1B), which are known to express mutant p53.
Cell density-dependent regulation of p73 transcription. To study the underlying mechanism of cell density-dependent regulation of p73, we next examined mRNA levels of p73 in cells at different densities using RT-PCR. Isoform-specific primers were used to detect TAp73 and $\Delta \mathrm{Np} 73$. As shown in Fig. 3A, TAp73 RNA levels increased with increasing density, which is parallel to the protein pattern of p73a (Fig. 2A). Since it was reported that $\Delta N p 73$ mRNA and protein levels were higher in confluent cells than at low density (20), we also examined the mRNA levels of $\Delta \mathrm{Np} 73$. However, our data demonstrated that $\Delta \mathrm{Np} 73$ levels were basically unchanged at different cell densities.

To verify the PCR results and evaluate TAp73 promoter activity at different densities, we established a stable MCF-7 subline transfected with a reporter plasmid carrying TAp73 promoter from -2713 to +20 , which was named MCF-7/ p73PF/Luc. It has been confirmed that the reporter gene in the clone used for the following study is not affected by the positional effect (data not shown). Results from luciferase assays on $\mathrm{p} 73$ promoter activity in $\mathrm{MCF}-7 / \mathrm{p} 73 \mathrm{PF} / \mathrm{Luc}$ at different density indicate that $\mathrm{p} 73$ promoter activation increases with cell density, which is consistent with the variation of TAp73 mRNA levels (Fig. 3). These results suggest that high levels of $\mathrm{p} 73 ß$ at low density are independent of p73 transcription. In contrast, concomitant changes in p73 $\alpha$ protein levels, E2F-1, TAp73 mRNA levels suggest that transcriptional regulation plays a critical role in cell density-dependent regulation of $\mathrm{p} 73 \alpha$.

p73ß protein stability is cell density-dependent. To test whether modulation of $\mathrm{p} 73 \beta$ stability was involved in cell density-dependent regulation of $\mathrm{p} 733, \mathrm{MCF}-7$ cells at low $\left(2 \times 10^{4}\right)$ and higher density $\left(2 \times 10^{5}\right)$ were treated with the protein synthesis inhibitor cycloheximide (CHX) in a timecourse experiment (for $0 \mathrm{~min}, 45 \mathrm{~min}, 1.5,3$ and $5 \mathrm{~h}$ ) and p73ß protein levels were analyzed by Western blotting. The results show that $\mathrm{p} 73 \mathrm{~B}$ was relatively stable in cells at low density, whereas its degradation in cells at higher density was faster (Fig. 3B). This suggests that modified protein stability may contribute to lower protein levels of $\mathrm{p} 73 \beta$ at higher densities.

Cell density-dependent regulation of $p 73 \beta$ is independent of ubiquitin ligase AIP4/Itch. AIP4/Itch is a human Hectubiquitin protein ligase (E3) that binds to p73 and targets it to proteasome-dependent degradation (21). AIP4/Itch expression is downregulated upon DNA damage, thereby allowing stabilization of the p73 protein (16). To test whether AIP4/ Itch-mediated regulation of p73 contributes to cell densitydependent modulation of $\mathrm{p} 733$, we first tested whether the expression of AIP4/Itch is cell density-dependent. As shown in Fig. 4A, AIP4/Itch protein levels increased in a densitydependent manner, which are inversely correlated with $\mathrm{p} 733$ expression. We next examined whether knocking down AIP4 using AIP4-specific siRNA modulate $\mathrm{p} 733$ protein levels at a relatively low density. As shown in Fig. 4B, AIP4 siRNA effectively inhibited the expression of AIP4 in the transfected cells. Interestingly, AIP4 knockdown results in increase in p $73 \alpha$ levels but little change in p $73 ß$ levels. Although the experiments in Fig. 3B suggest a correlation between lower 
A

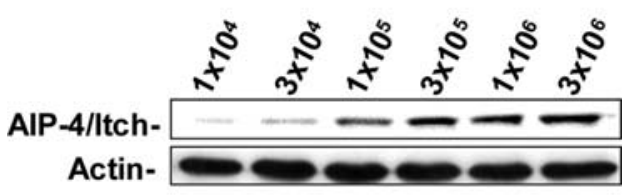

B

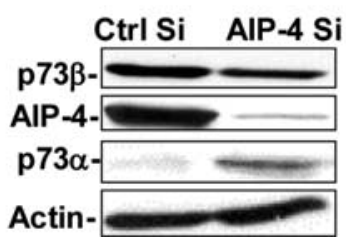

Figure 4. (A) AIP-4/Itch levels increased with increasing culture density MCF-7 were inoculated at different densities as indicated. AIP-4 protein levels were determined by Western blotting. (B) Knockdown of AIP-4 in high density cells did not increase p73ß protein levels. AIP-4 was knocked down in MCF-7 cells using AIP-4-specific siRNA. AIP-4 and p73 levels in control siRNA and AIP-4 siRNA expressing MCF-7 cells were determined by Western blot using anti-p73 $\alpha$ (H79) and anti-p73ß (AB3) antibodies.

levels of p73ß and decreased protein stability at higher density, results from AIP4 knockdown do not support the role of AIP4 in p73ß degradation at higher density.

Subcellular localization of p73 in MCF-7 cells at different densities. To verify the differential expression of $\mathrm{p} 733$ at different cell densities, we examined $\mathrm{p} 73 ß$ expression by immunocytochemistry. To this end, MCF-7 cells were inoculated in chamber slide at different density followed by immunostaining. The results show that p73ß signal was very strong in cells at low density but weak in cells at higher density (Fig. 5A), which is consistent with the Western blot data. While examining p73 expression, we noticed that high levels of p73ß in cells at lower density were primarily located in the nucleus. In contrast, substantial p73ß in the cells at higher density appeared to be cytosolic. To further investigate this issue, we examined p73ß localization in MCF-7 cells at different density by cell fractionation. As shown in Fig. 5B, preparation of nuclear and cytosolic fractions was successful, as demonstrated by distinctive signals of lamin $\mathrm{B}$ and actin, which are enriched in nucleus and cytosol, respectively. Clearly, as shown in Fig. 5B, p73 protein levels decreased with increasing cell density. Interestingly, however, a different pattern of p73 expression was observed in low versus high density cultures. In low density cultures, p73 was found mainly in nucleus whereas in dense cultures, p73 was predominantly cytosolic.

\section{Discussion}

In this study, we investigated the effect of variation in cell densities on p73 expression in breast cancer cells. We demonstrate that $\mathrm{p} 73$ levels, in particular $\mathrm{p} 73 \beta$ protein levels, are sensitive to changes in cell density. This suggests that cell-cell interaction and extra cellular matrix-mediated signaling, which are involved in cell density-dependent regulation and in tumor microenvironment modulation, may affect

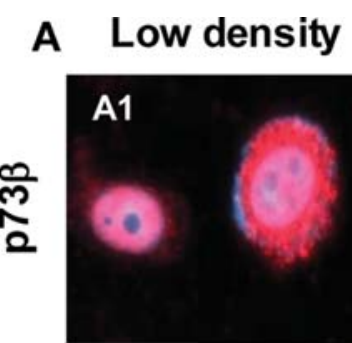

\section{High density}
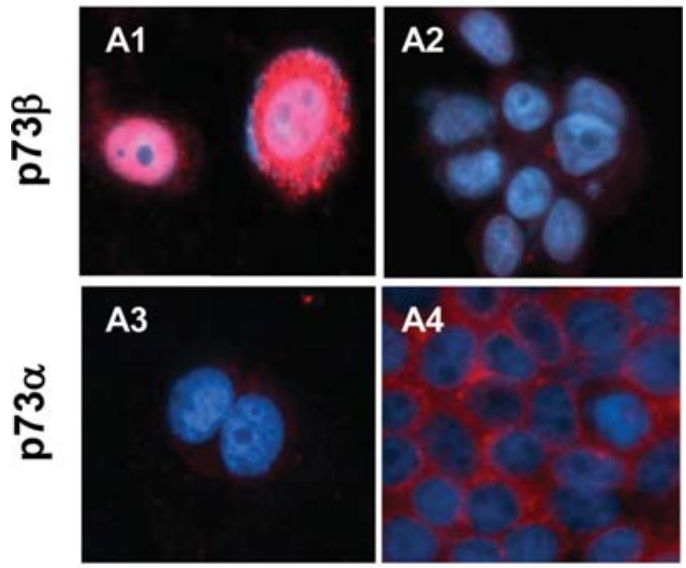

B

Nuclear Cytosolic
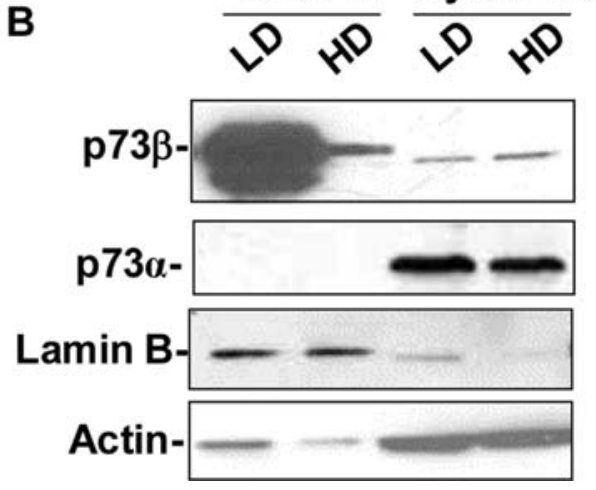

Figure 5. Subcellular localization of p73 changes in MCF-7 cells in a density-dependent manner. MCF-7 cells were cultured at indicated densities in 8-well coverglass chamber slides for $48 \mathrm{~h}$. After $48 \mathrm{~h}$, cells were fixed and immunostained for p73 using anti-p73 $\alpha$ (C24) and anti-p73ß (AB3) antibodies and FITC-labeled secondary antibody. Images were taken using (Carl-Zeiss) confocal microscope. (B) MCF-7 cells were cultured at low $\left(2 \times 10^{4}\right)$ and high $\left(2 \times 10^{6}\right)$ densities in 60 -mm dishes. After $48 \mathrm{~h}$ of incubation, cells were collected. Nuclear and cytosolic fractions were prepared, and subjected to Western blot analysis using anti-p73 (AB3 and C24), antilamin B, and anti-actin antibodies.

p73 expression and function. This finding identified a novel mechanism that regulates p73 expression and may advance our understanding of p73 expression in human cancer tissues.

In the characterization of cell density-dependent regulation of p73, we found that p73 isoforms are differentially regulated by cell density. In contrast to $\mathrm{p} 73 \alpha$, whose levels increase with cell densities, p73ß protein levels are high at low density and decrease with density increase. This suggests that in addition to alternate promoter sets and splicing (2), cell density-related factors may play a role in the differential regulation of $\mathrm{p} 73$ isoforms. In this study, identification of p $73 \alpha$ and p73ß was mainly based on established antibodies primarily recognizing specific isoforms. Although this may have certain limits in explaining other isoforms, evidence supporting the notion that cell density differentially regulates p73 isoforms is concrete. The effect of density-dependence of other p73 isoforms and more specific approaches that discerns individual forms will be tested in future studies.

The mechanisms of cell density-dependent regulation of p73 appear to be complicated. Cell density may regulate 
p73 $\alpha$ and p73ß levels by different mechanisms. Our data showed that density-dependent regulation of p73 was p53independent, although p53 levels also changed with cell densities. Since p $73 \alpha$ levels behaved concomitantly with E2F-1 levels and TAp73 mRNA levels in response to density variation, cell density-dependent regulation of $\mathrm{p} 73 \alpha$ is mainly regulated at the transcriptional level. This is consistent with a recent report that p73 may be induced in confluent cells with pRB pathway alterations (22). In contrast, p73ß behaved oppositely with p73 $\alpha$, E2F-1 and TAp73 mRNA, suggesting a different mechanism. Indeed, results from CHX blocking experiments suggest that $\mathrm{p} 73 ß$ protein at low density is more stable than at high density. However, knockdown of AIP4/ Itch, a ubiquitin ligase that regulates p73 degradation (16), in MCF-7 cells only resulted in increased protein levels of p73 $\alpha$ but not p73ß (Fig. 4). As p73 stability could be regulated by other factors, such as promyelocytic leukemia (PML) (23), how $\mathrm{p} 73 ß$ is regulated in density-dependent manner requires further investigation.

Given the differential regulation of p73 isoforms associated with cell density, the functional relevance of a specific isoform appears to be complicated. Relatively, cell densitydependent regulation of $\mathrm{p} 73 \beta$ was more evident than $\mathrm{p} 73 \alpha$ (Fig. 2). Previous reports suggest that $\mathrm{p} 73 ß$ was more potent than $\mathrm{p} 73 \alpha$ in regulating p53-dependent genes $(3,24)$. Based on our data of cell density-dependent proliferation (Fig. 1D), high levels of p $73 ß$ at very low density were associated with slower proliferation. This is consistent with the common observations that cells do not grow well under too sporadic conditions. More specific designs correlating p73 isoforms and cellular functions at different densities will be tested in future studies.

Immunostaining experiments indicate that subcellular localization of p73 isoforms changes with changes in cell density. Many signaling proteins are regulated by alterations in their subcellular location. MDM2 is known to alter subcellular localization of p73 (25). Differential regulation of $\mathrm{p} 73 \alpha$ and $\mathrm{p} 73 ß$ cellular localization at different culture densities adds an important aspect to be considered while manipulating p73-dependent gene expression for tumor cell growth inhibition and apoptosis. As in all other p73-related studies, differentiation among p73 isoforms is a complicated issue. In this study the differential regulation of p73 isoforms was mainly based on antibodies primarily recognizing p73 $\alpha$ or $B$. We acknowledge the limit of this approach in explana-tion of other p73 isoforms. Nevertheless, findings in this report are intriguing and may lead to more studies on $\mathrm{p} 73$ regulation by cell density and extra cellular matrix.

In summary, our data demonstrate an interesting finding that $p 73 \alpha$ and $p 73 \beta$ levels are differentially regulated in a cell density-dependent manner. In particular, expression of p73ß was more sensitive to cell density. Mechanistic studies suggest that cell density-dependent regulation of $\mathrm{p} 73 ß$ mainly occurs at the protein level. In contrast, the regulation of $73 \alpha$ appears to be at the transcriptional level. Moreover, regulation of p73ß was independent of p53 and AIP4/Itch, although involvement of other factors requires further exploration. Our results suggest that cell-cell, cell-matrix interactions, which are critical for tumor initiation and invasion, may play a role in regulating p73 expression. More detailed studies are required to identify specific molecules involved in intercellular interactions that are responsible for density-mediated regulation of p73 expression in breast cancer.

\section{Acknowledgements}

This work was supported by a Seed Grant to X.Y. from the University of Oklahoma Cancer Center Institutional Research Grant from the American Cancer Society ASC-IRG \#IRG05-066-01; X.Y. is also supported by a Health Research Grant from Oklahoma Center for Advancement of Science and Technology (HR07-108) and a Research Scholar Grant from the American Cancer Society (RSG-08-138-01-CNE).

\section{References}

1. Arrowsmith $\mathrm{CH}$ : Structure and function in the p53 family. Cell Death Differ 6: 1169-1173, 1999.

2. Melino G, De Laurenzi V and Vousden KH: p73: Friend or foe in tumorigenesis. Nat Rev Cancer 2: 605-615, 2002.

3. Ueda Y, Hijikata M, Takagi S, Chiba T and Shimotohno K: New p73 variants with altered C-terminal structures have varied transcriptional activities. Oncogene 18: 4993-4998, 1999.

4. Stiewe T and Putzer BM: Role of p73 in malignancy: tumor suppressor or oncogene? Cell Death Differ 9: 237-245, 2002.

5. Gong L, Li Y, Nedeljkovic-Kurepa A and Sarkar FH: Inactivation of NF-kappaB by genistein is mediated via Akt signaling pathway in breast cancer cells. Oncogene 22: 4702-4709, 2003.

6. Yuan ZM, Shioya H, Ishiko T, et al: p73 is regulated by tyrosine kinase c-Abl in the apoptotic response to DNA damage. Nature 399: 814-817, 1999.

7. Seelan RS, Irwin M, van der Stoop P, Qian C, Kaelin WG Jr and Liu W: The human p73 promoter: characterization and identification of functional E2F binding sites. Neoplasia 4: 195-203, 2002.

8. Garrett SD, Lee HA, Friar PM and Morgan MR: Validation of a novel estrogen receptor-based microtitration plate assay for the determination of phytoestrogens in soy-based foods. J Agric Food Chem 47: 4106-4111, 1999.

9. Kartasheva NN, Contente A, Lenz-Stoppler C, Roth J and Dobbelstein M: p53 induces the expression of its antagonist p73 Delta N, establishing an autoregulatory feedback loop. Oncogene 21: 4715-4727, 2002.

10. Vossio S, Palescandolo E, Pediconi N, Moretti F, Balsano C, Levrero $\mathrm{M}$ and Costanzo A: DN-p73 is activated after DNA damage in a p53-dependent manner to regulate p53-induced cell cycle arrest. Oncogene 21: 3796-3803, 2002.

11. Stiewe T and Putzer BM: Role of the p53-homologue p73 in E2F1-induced apoptosis. Nat Genet 26: 464-469, 2000.

12. Watanabe K, Ozaki T, Nakagawa T, et al: Physical interaction of p73 with c-Myc and MM1, a c-Myc-binding protein, and modulation of the p73 function. J Biol Chem 277: 15113-15123, 2002.

13. Das S, El-Deiry WS and Somasundaram K: Regulation of the p53 homolog p73 by adenoviral oncogene E1A. J Biol Chem 278: 18313-18320, 2003.

14. Zeng X, Li X, Miller A, et al: The N-terminal domain of p73 interacts with the $\mathrm{CH} 1$ domain of $\mathrm{p} 300 / \mathrm{CREB}$ binding protein and mediates transcriptional activation and apoptosis. Mol Cell Biol 20: 1299-1310, 2000.

15. Agami R, Blandino G, Oren M and Shaul Y: Interaction of c$\mathrm{Abl}$ and p73alpha and their collaboration to induce apoptosis. Nature 399: 809-813, 1999.

16. Distefano M, Scambia G, Ferlini C, et al: Anti-proliferative activity of a new class of taxanes (14beta-hydroxy-10-deacetylbaccatin III derivatives) on multidrug-resistance-positive human cancer cells. Int J Cancer 72: 844-850, 1997.

17. Nozell S, Wu Y, McNaughton K, Liu G, Willis A, Paik JC and Chen X: Characterization of p73 functional domains necessary for transactivation and growth suppression. Oncogene 22: 4333-4347, 2003.

18. Costanzo A, Merlo P, Pediconi N, et al: DNA damagedependent acetylation of $\mathrm{p} 73$ dictates the selective activation of apoptotic target genes. Mol Cell 9: 175-186, 2002. 
19. Chen X, Zheng Y, Zhu J, Jiang J and Wang J: p73 is transcriptionally regulated by DNA damage, $\mathrm{p} 53$, and $\mathrm{p} 73$. Oncogene 20 : 769-774, 2001

20. Waltermann A, Kartasheva NN and Dobbelstein M: Differential regulation of p63 and p73 expression. Oncogene 22: 5686-5693, 2003.

21. Rossi M, De Laurenzi V, Munarriz E, et al: The ubiquitinprotein ligase Itch regulates p73 stability. EMBO J 24: 836-848, 2005.

22. Beitzinger M, Hofmann L, Oswald C, et al: p73 poses a barrier to malignant transformation by limiting anchorage-independent growth. EMBO J 27: 792-803, 2008.
23. Oberst A, Rossi M, Salomoni P, Pandolfi PP, Oren M, Melino G and Bernassola F: Regulation of the p73 protein stability and degradation. Biochem Biophys Res Commun 331: 707-712, 2005.

24. De Laurenzi V, Costanzo A, Barcaroli D, et al: Two new p73 splice variants, gamma and delta, with different transcriptional activity. J Exp Med 188: 1763-1768, 1998.

25. Gu J, Nie L, Kawai H and Yuan ZM: Subcellular distribution of p53 and p73 are differentially regulated by MDM2. Cancer Res 61: 6703-6707, 2001 